\title{
Effect of Food/Beverage and Selected Drugs on the Oral Absorption of Artemether-Lumefantrine Tablet: an in situ and in vivo Study
}

\author{
Original \\ Sunday O. Awofisayo', Matthew I. Arhewoh ${ }^{2}$ and Augustine O. Okhamafe ${ }^{2}$ \\ Article \\ ${ }^{1}$ Department of Clinical Pharmacy and Biopharmacy, Faculty of Pharmacy, University of \\ Uyo, Nigeria. \\ ${ }^{2}$ Department of Pharmaceutics and Pharmaceutical Technology, Faculty of Pharmacy, \\ University of Benin, Benin City, Nigeria.
}

\begin{abstract}
Aim: This study aims at assessing the effect of some meals/beverage and selected drugs on oral absorption of artemether (ATM) and lumefantrine (LMF).

Methods: In the in situ model, artemether-lumefantrine (AL) tablets were crushed and administered to anesthetized rabbits $(n=2)$ via oral cannula either alone (CTR1) or with food components/beverage [i.e., starch (STC), albumin (ALB), sunflower oil (SFO) or carbonated drinks (CBS)] or drugs [i.e., lamivudine (LMV) or metronidazole (MTN)]. Blood samples were taken from cannulated carotid artery post dose administration. In the in vivo model, forty two healthy human subjects (28 male and 14 female) in groups of six persons received AL tablet alone (CRT2) or "eba" (cassava starch) with melon soup (EMS) or corn pap with milk and "akara" (fried beans cake) (PMA) or fruits (FTS) or CBS or drugs (i.e., LMV or MTN). ATM and LMF plasma concentrations were obtained simultaneously from plasma using reverse phase high pressure liquid chromatographic analysis.

Results: There was significant reduction in ATM Ka due to STC, ALB, CBS, MTN and LMV $\left(\mathrm{Ka} \leq 1.371 \mathrm{~h}^{-1}\right)$ compared with CRT1 (3.567 $\left.\left.\mathrm{h}^{-1}\right), p<0.05\right)$. LMV and MTN also reduced the Ka and AUC of ATM and LMF, $p<0.05$. Similarly, in vivo study showed significantly lower ATM AUC and Ka values for EMS $\left(0.775 \mu \mathrm{ghmL}^{-1}\right.$ and $\left.0.041 \mathrm{~h}^{-1}\right)$ and CBS $\left(0.248 \mu \mathrm{ghmL}^{-1}\right.$ and $\left.4.155 \mathrm{~h}^{-1}\right)$ compared with the CRT2 $\left(6.090 \mu \mathrm{ghmL}^{-1}\right.$ and $\left.0.362 \mathrm{~h}^{-1}\right)$.

Conclusion: EMS or CBS resulted in significant reduction in the bioavailability of ATM and LMF and can influence the treatment outcomes.
\end{abstract}

Received: 18 January, 2019, Accepted: 29 June, 2019.

Key Words: Artemether-Lumefantrine; Carbonated beverage, Food components, Lamivudine, Metronidazole.

Corresponding Author: Sunday O. Awofisayo, Department of Clinical Pharmacy and Biopharmacy, University Of Uyo, Post Office Box 4257, Uyo 520003, Nigeria, Tel.: +2348037947338, E-mail: sundayawofisayo@uniuyo.edu.ng.

Bulletin of Faculty of Pharmacy, Cairo University, ISSN: 1110-0931, Vol. 57, No. 2

\section{INTRODUCTION}

Malaria is a disease of great concern the world over with at least 300 to 500 million people diagnosed of the illness yearly ${ }^{[1]}$. It is considered a major cause of morbidity and mortality in sub-Saharan Africa affecting children below the age of 5 years and pregnant women $^{[2,3]}$. Artemether-lumefantrine (AL) is one of the fixed-dose artemisinin-based combination therapies (ACT) that was recommended as a first line treatment for uncomplicated P. falciparum malaria worldwide ${ }^{[4-6]}$. Oral formulations of AL of various strengths are available as tablets (for adults) and dispersible (for children) $)^{[7-9]}$. The design of fixed-dose combinations (FDCs) of antimalarial agents to combat the menace posed by malaria has been widely accepted and adopted as national health policy in many countries ${ }^{[10-12]}$.

The administration of AL with other medications becomes inevitable with the co-morbidities of malaria and infections caused by viruses, bacteria or protozoan. Reports have shown that Human Immunodeficiency
Virus (HIV) and malaria infections have overlapping geographical distribution ${ }^{[13]}$. The two diseases have been reported to cause annually about 4 million deaths worldwide ${ }^{[14,15]}$. Drugs such as 5-nitroimidazole agents [i.e., metronidazole (MTN)] and nucleoside reverse transcriptase inhibitor [i.e., lamivudine (LMV)] used in amoebiasis and HIV treatments, respectively have been prescribed and ingested with AL tablet. There is limited evidence of drug-drug interaction ensuing from the oral intake of drugs that can affect AL safety in Africa. Oral absorption of drugs is a complex mechanism ${ }^{[16,17]}$. Following up with this fact, in an acute malarial condition, sufferers are usually anorexic, pyretic and often feel like vomiting ${ }^{[17]}$. This can be so severe that several meal types may be unappealing to the sick person and generally there is significant loss of appetite. Consequently, the type and volume of meals taken in this condition may vary widely. Some antimalarial drugs have been reported to possess improved absorption when ingested with fatty meals [e.g., halofantrine and lumefantrine $(\mathrm{LMF})]^{[17-20]}$. In some parts of Africa, specifically 
Eastern Nigeria, there is the traditional/cultural belief that oil intake is contributory to malaria infection $^{[16]}$. Premji and co-workers in Uganda reported that African meals consisted of cereals (maize, millet, sorghum and rice) and the starchy roots (potatoes, sweet yam, yam and cassava) ${ }^{[17]}$.

This study aimed at assessing the effect of common meal types and co-prescribed drugs taken with $\mathrm{AL}$ on oral absorption of artemether (ATM) and LMF in healthy subjects.

\section{MATERIALS AND METHODS}

\section{Experimental animals}

New Zealand albino male rabbits weighing $(1.6-2.0 \mathrm{~kg})$ were obtained from the animal facility (University of Lagos animal house). Animals (14) were maintained at $25 \pm 2{ }^{\circ} \mathrm{C}$ and relative humidity of $55 \pm 5 \%$ and $12 \mathrm{~h} \mathrm{light/dark}$ cycle with free access to water. The protocol of the study was approved by the Committee for Use of Laboratory Animals of the Faculty of Pharmacy, University of Uyo, Nigeria with approval reference number FUP021016. Animals were anesthetized with intra-peritoneal injection of urethane chloralis $0.5 \mathrm{ml} / \mathrm{kg}$ body weight. The animals were also given a tracheal bypass using a cannula. The carotid arteries were cannulated using a 16-18 gauge catheter to aid successive sample collection. Animals were handled based on the American Psychological Associations Guidelines for Ethical Conduct in the Care and Use of Non-human Animals in Research ${ }^{[21]}$.

\section{Chemicals}

Acetonitrile, potassium dihydrogen phosphate, methanol and tetrahydrofuran (THF) and all other reagents were of analytical grade, products of Sigma Aldrich, Germany. AL tablet, Coartem ${ }^{\circledR}$ was a product of Novartis, France. MTN (Loxagyl $\AA$, May and Baker, Nigeria) and LMV (Lami-150®, McNeil and Argus, India) were purchased in a pharmacy.ATM and LMF (reference standard) and halofantrine (internal standard) powdered products were donated by Central Research Laboratory (CRL), University of Lagos. Details of samples used in the study are presented in (Table 1).

Table 1: Details of the food components and drugs used in the study

\begin{tabular}{|c|c|c|c|}
\hline $\begin{array}{l}\text { Name of food } \\
\text { component/ } \\
\text { drugs }\end{array}$ & $\begin{array}{l}\text { Manufacturing } \\
\text { Date }\end{array}$ & Expiry Date & Origin \\
\hline Albumin & 01-2014 & $12-2016$ & $\begin{array}{l}\text { Alpha Chemika } \\
\text { India }\end{array}$ \\
\hline Starch & $05-2013$ & $05-2017$ & $\begin{array}{l}\text { Park Services } \\
\text { Ukraine }\end{array}$ \\
\hline Sunflower oil & 012012 & 01-2017 & $\begin{array}{l}\text { JiannHairu } \\
\text { China }\end{array}$ \\
\hline $\begin{array}{l}\text { Carbonated } \\
\text { drink }\end{array}$ & $05-2014$ & $11-2015$ & $\begin{array}{l}\text { Coca-cola } \quad \text { Co } \\
\text { Nigeria }\end{array}$ \\
\hline Coartem & 07-2014 & 06-2016 & $\begin{array}{l}\text { Novartis Pharm. } \\
\text { Corp USA }\end{array}$ \\
\hline Loxagyl & 01-2014 & 01-2016 & $\begin{array}{l}\text { May and Baker, } \\
\text { Nigeria }\end{array}$ \\
\hline Lami-150 & $05-2014$ & 04-2016 & $\begin{array}{l}\text { McNeil and } \\
\text { Argus; India }\end{array}$ \\
\hline
\end{tabular}

\section{Dosing of animals and collection of samples}

A single dose of AL was administered to rabbits at 10.7 $\mathrm{mg} / \mathrm{kg}$ body weight with reference to the LMF content. Food components (equivalent to $40 \mathrm{mg} / \mathrm{kg}$ body weight) or drugs (at 3.75 and $10 \mathrm{mg} / \mathrm{kg}$ LMV and MTN, respectively) were similarly administered along with AL based on 10.7 $\mathrm{mg}$ dosing with respect to LMF content $/ \mathrm{kg}$ animal body weight, through oral cannula delivered down into the esophagus. Samples were collected through an in-dwelling cannula inserted through the carotid artery. Blood samples (1-2 $\mathrm{mL}$ ) were taken at $0,1,2,4$ and $6 \mathrm{~h}$ post-administration of $\mathrm{AL}$ alone or with food components or drugs. The samples were deposited into heparinized propylene bottles and thereafter centrifuged (ultra-centrifuge, USA) for 5 min. The plasma fraction was subsequently obtained and stored pending analysis.

\section{Study site and ethical approval for human experiment}

The research was conducted at the Department of Clinical Pharmacy and Biopharmacy, Faculty of Pharmacy, University of Uyo, Nigeria. The study protocol was approved by the Institutional and Ethics Review Board of University of Uyo Teaching Hospital with approval reference number UUTH/102016.

\section{Clinical study design}

This was a non-randomized, single dose, single center pharmacokinetic study. The study was conducted according to Good Clinical Practice. To ensure confidentiality, identification codes were used.

\section{Screening of volunteers}

Recruitment into the study was done through sensitization meetings conducted within the premises of University of Uyo, among selected staff and students, in the University community. Permission to conduct the awareness meetings was obtained from the University Administration. Interested participants were registered and invited to attend a briefing where detailed clarification about the study and its objectives were discussed. The potential inconveniences and adverse events were carefully explained. Potentially eligible participants were identified and listed for screening.

\section{Clinical study design}

All the potential subjects that turned up for the screening exercise were examined. The baseline screening was based on the inclusion and exclusion criteria to get eligible participants that were enrolled in the study. Baseline screening included taking medical history, examining physical and medical parameters (i.e., vital signs) and 
blood laboratory tests (i.e, parasitology for detecting malaria parasites in blood smear, haematology for full blood count) along with biochemistry [i.e., urea, creatinine, alanine transaminase (ALT), aspartate transaminase (AST) and alkaline phosphatase (ALP)]. The exclusion criteria included smoking, habitual alcohol intake, pregnancy, any known renal or hepatic dysfunction and taking of antimalarial drugs within the space of 2 months before the screening period. Volunteers were also excluded if they had ascites, peptic ulcer or other known gastrointestinal diseases. Volunteers were eligible to participate in the study if they had a negative result for malaria parasite test (using microscopic detection of parasites in blood smear after Giemsa stain).

\section{Clinical study design}

All the eligible volunteers who fulfilled the conditions of the inclusion and exclusion criteria were enrolled. Out of 54 subjects registered for the screening, 42 were enrolled into the study and were subsequently advised in readiness for the study (i.e., abstinence from drugs and alcohol).

\section{Subject management}

Forty two healthy Nigerian volunteers, aged 24 to 35 years were enrolled in the study. The subjects were randomly assigned to seven groups (4 male and 2 female per group) and each group assigned to one treatment type. The study was conducted in accordance with the guidelines of the declaration of Helsinki and Good Clinical Practices Guidelines set up by the International Conference on Harmonization and local applicable laws and regulations ${ }^{[20]}$.

\section{Drug administration and blood sample collection}

The volunteers in each group received a single dose of AL $(80 / 480 \mathrm{mg})$ tablet administered with $0.5 \mathrm{~L}$ of water after eating a particular meal/beverage [i.e.,"eba"(cassava starch meal) with melon soup (EMS) or corn pap with milk and "akara" (fried bean cake) (PMA) or fruits (FTS) or carbonated drink (CBS)]or ingesting either of the drugs (i.e. MTN or LMV) after an overnight fast. One of the group served as control (CTR1) ingesting AL alone. Food and water intake was restricted for 4 hours after dosing. Blood samples $(5 \mathrm{~mL})$ were taken at $0,1,2,4,6$ and 8 hours post-dose from an indwelling intravenous cannula inserted into the upper arm and deposited into lithium heparinized tubes. The samples were centrifuged at 4000 $\mathrm{rev} / \mathrm{min}$ for $5 \mathrm{~min}$ and the plasma fractions transferred to plain polypropylene tubes. This was stored until analysis.

\section{Reference solution preparation}

The internal standard (IS) solution of halofantrine was prepared by transferring $10 \mathrm{mg}$ into $10 \mathrm{~mL}$ volumetric flask. A volume of $6 \mathrm{~mL}$ of methanol was added to dissolve and subsequently made to mark by adding methanol to produce stock concentration of $1 \mathrm{mg} / \mathrm{mL}$. A weight of $20 \mathrm{mg}$ and $120 \mathrm{mg}$ of reference standard ATM and LMF, respectively were transferred into different $10 \mathrm{~mL}$ volumetric flasks. These were dissolved in acetonitrile and THF, to produce $2 \mathrm{mg} / \mathrm{mL}$ and $12 \mathrm{mg} / \mathrm{mL}$ stock solutions, respectively. Mixed standard solutions of AL and IS were prepared by adding equal volumes of ATM and LMF stock solutions $(2 \mathrm{~mL})$ and dispensed into $5 \mathrm{~mL}$ plain polypropylene sample bottles to produce $1 \mathrm{mg} / \mathrm{mL}$ and 6 $\mathrm{mg} / \mathrm{mL}$ of the standards, respectively. Serial dilutions of the mixed standards and reference standard solutions were made to produce graded concentrations from $0.01 / 1.0$ to 20.0/100.0 mg/mL using acetonitrile and THF (50:50, \% $\mathrm{v} / \mathrm{v})$ to dilute the mixed standard solutions. The obtained solutions were spiked with IS stock solution to give $5 \mu \mathrm{g} / \mathrm{mL}$ with micropipette.

\section{Sample analyses}

The plasma samples were processed at Central Research Laboratory of the University of Lagos, Nigeria for quantification of the artemether and lumefantrine in plasma. ATM and LMF were extracted from plasma using solid phase extraction method (Oasis HLB micro-elution plate, waters, Milford, USA). The concentrations of ATM and LMF for the various samples were determined using reverse phase high pressure liquid chromatographic (RPHPLC) system (Chemstation, Germany) with Zorbact Eclipse XDB 150 × 4.6 × $5 \mu \mathrm{m}$ column. Mobile phase consisted of acetonitrile: potassium dihydrogen phosphate $(70: 30, \% \mathrm{v} / \mathrm{v})$ and flow rate and wavelength of detection of $1.0 \mathrm{~mL} / \mathrm{min}$ and $216 \mathrm{~nm}$, respectively at $25^{\circ} \mathrm{C}^{[22]}$. The peak area ratio for ATM and LMF, with respect to halofantrine, were deduced from the chromatogram while the drug concentrations of the analytes were obtained from a standard calibration curve. ATM and LMF peak ratio for each time collection were computed to obtain the plasma levels of the component drugs (i.e., ATM and LMF).

\section{Pharmacokinetic analysis}

APK pharmacokinetic software (RxKinetics, USA)and GraphPad Prism (USA) were employed for the computation of the oral absorption profile of ATM and LMF. A first order rate kinetics was assumed. The maximum plasma concentration (Cmax), absorption rate constant (Ka), fraction absorbed (F) and total drug absorbed (AUC) were obtained for each subject and the mean values for the dosing groups computed.

\section{Statistical analysis}

Data were analyzed using Minitab for windows statistical package version 12 (Minitab, Inc, USA). The statistical analysis employed single factor analysis of variance (ANOVA) of the means of parameters for the dosing condition and tested at $95 \%$ confidence interval (CI). 


\section{RESULTS AND DISCUSSION}

\section{In situ study}

The regression coefficients for ATM and LMF for the analysis during the calibration were 0.9997 and 0.9996 , respectively. The plasma concentrations versus time for ATM and LMF in the respective treatments are presented in Figures 1-3, while their pharmacokinetic parameters are summarized in Table 2. The bioavailability of ATM was reduced in the presence of STC, ALB and CBS, $p<0.05$. SFO produced significantly higher ATM rate and extent of absorption compared with the other treatments, $p<0.05$. This study also revealed a higher $\mathrm{C}_{\max }$ for LMF in

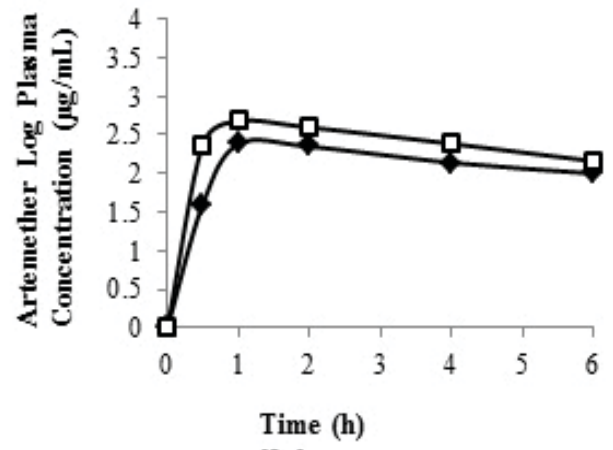

[la]

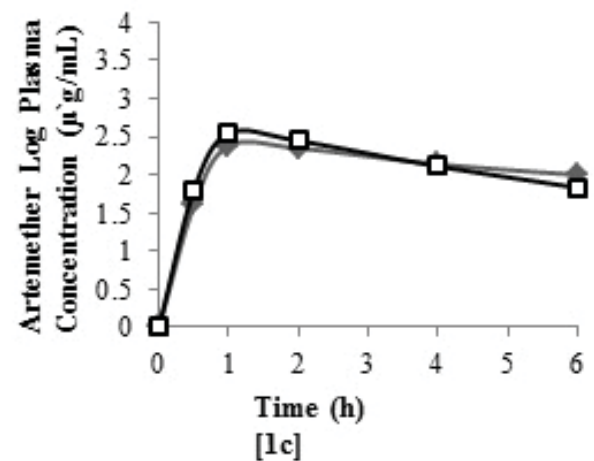

the presence of SFO compared with the other treatments, $p<0.05$ (Figures 1 and 2).

In Figure 2, there was no significant difference in the parameters for CTR1 compared with SFO for the actives in AL tablet. Similarly, Figure 2 presented the pharmacokinetic profiles for ATM and LMF in the presence of ALB based on the inference from the plasma concentration versus time plot. There was significant reduction in the parameters (i.e, $\mathrm{Ka}, \mathrm{C}_{\text {max }}$ and AUC) of ATM and LMF for ALB when compared with CTR1, $p<0.05$. Figure 3 revealed the profile of ATM and LMF absorption in the presence of LMV or MTN. Both drugs caused significant reduction in the pharmacokinetic parameters of ATM and LMF $(p<0.05)$.
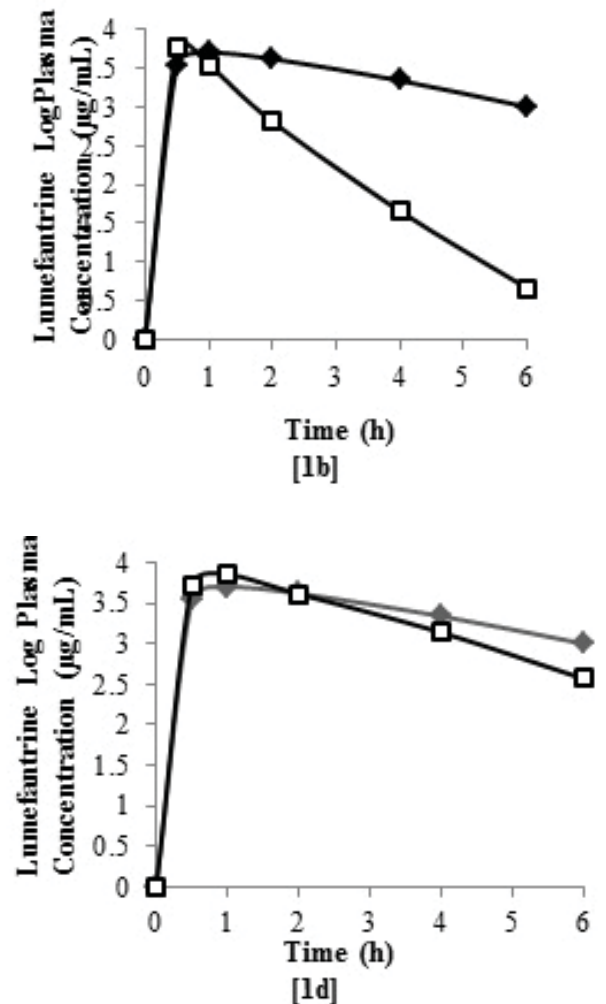

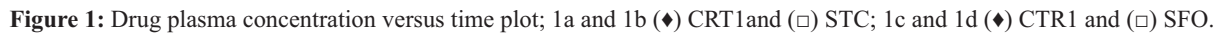




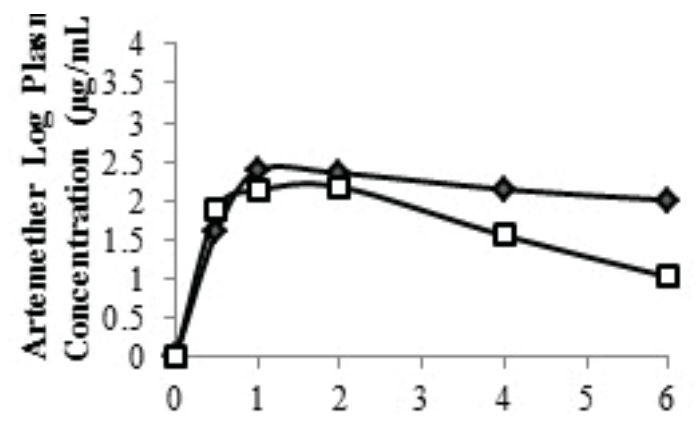

Time (h)

[2a]

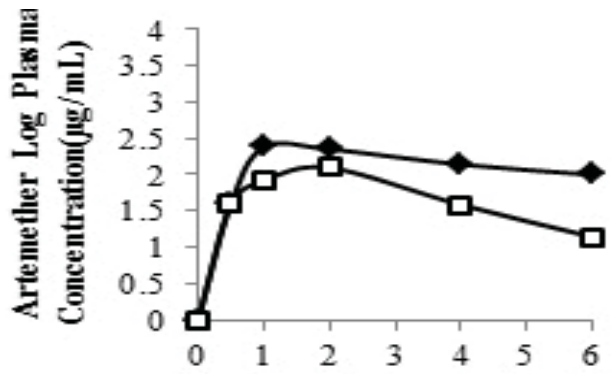

Time (h)

[2c]

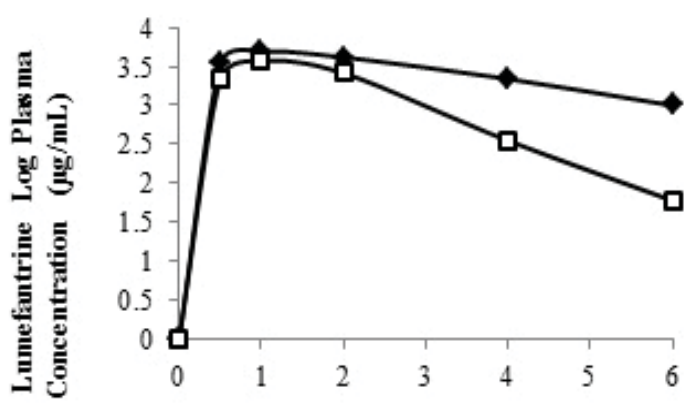

Time (h)

[2b]

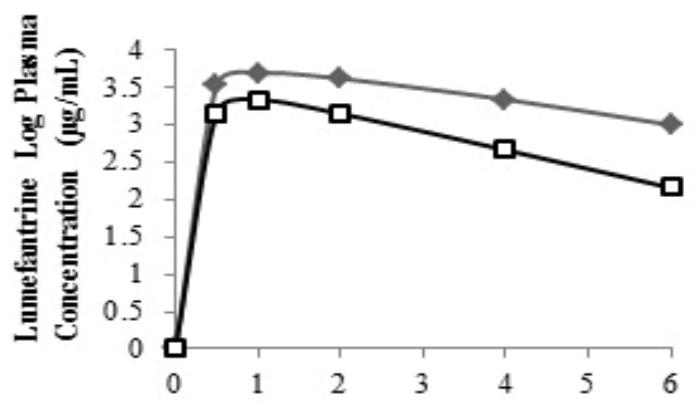

Time (h)

[2d]

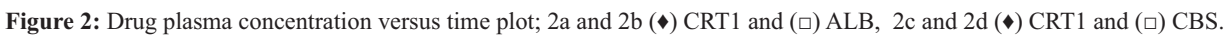
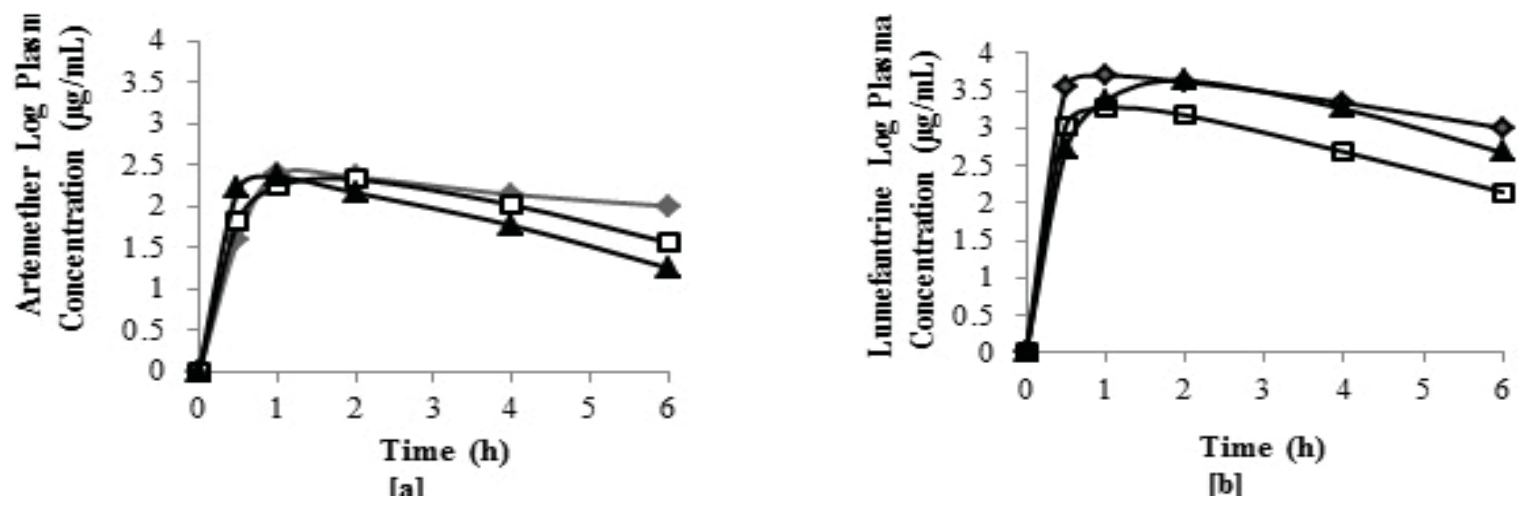

[b]

Figure 3: Drug plasma concentration versus time plot; 3a and 3b (•) CRT1, () LMV and (ム) MTN. 
ORAL ABSORPTION OF ARTEMETHER-LUMEFANTRINE

\begin{tabular}{|c|c|c|c|c|c|c|}
\hline Treatment & Drugs & $\mathrm{D}(\mathrm{mg})$ & $\mathrm{F}$ & $\mathrm{K}_{\mathrm{a}}\left(\mathrm{h}^{-1}\right)$ & $\mathrm{C}_{\max }(\mu \mathrm{g} / \mathrm{mL})$ & $\mathrm{AUC}(\mu \mathrm{g} . \mathrm{h} / \mathrm{mL})$ \\
\hline \multirow{2}{*}{ STC } & ATM & $14.830 \pm 4.200$ & $0.194 \pm 0.039$ & $1.371 \pm 0.051$ & $2.623 \pm 0.040$ & $14.417 \pm 0.225$ \\
\hline & LMF & $76.420 \pm 10.250$ & $0.159 \pm 0.022$ & $1.143 \pm 0.153$ & $3.523 \pm 0.106$ & $12.703 \pm 0.268$ \\
\hline \multirow{2}{*}{ SFO } & ATM & $25.970 \pm 12.020$ & $0.388 \pm 0.045$ & $3.217 \pm 0.231$ & $2.484 \pm 0.075$ & $12.648 \pm 0.648$ \\
\hline & LMF & $235.180 \pm 18.040$ & $0.490 \pm 0.038$ & $0.500 \pm 0.123$ & $3.843 \pm 0.050$ & $19.076 \pm 0.014$ \\
\hline \multirow{2}{*}{ ALB } & ATM & $49.640 \pm 19.960$ & $0.718 \pm 0.092$ & $1.233 \pm 0.259$ & $2.107 \pm 0.061$ & $10.359 \pm 0.556$ \\
\hline & LMF & $213.740 \pm 15.190$ & $0.445 \pm 0.031$ & $1.250 \pm 0.234$ & $3.555 \pm 0.066$ & $16.508 \pm 0.295$ \\
\hline \multirow{2}{*}{ CBS } & ATM & $46.550 \pm 7.390$ & $0.481 \pm 0.155$ & $1.330 \pm 0.321$ & $2.100 \pm 0.010$ & $10.023 \pm 0.009$ \\
\hline & LMF & $40.440 \pm 2.470$ & $0.084 \pm 0.049$ & $0.700 \pm 0.085$ & $3.321 \pm 0.097$ & $17.040 \pm 0.023$ \\
\hline \multirow{2}{*}{ LMV } & ATM & $50.530 \pm 21.110$ & $0.710 \pm 0.131$ & $1.697 \pm 0.253$ & $2.315 \pm 0.100$ & $11.675 \pm 0.226$ \\
\hline & LMF & $402.810 \pm 20.210$ & $0.849 \pm 0.050$ & $1.007 \pm 0.060$ & $3.253 \pm 0.068$ & $16.343 \pm 0.191$ \\
\hline \multirow{2}{*}{ MTN } & ATM & $38.810 \pm 6.060$ & $0.410 \pm 0.069$ & $0.720 \pm 0.221$ & $2.302 \pm 0.053$ & $11.505 \pm 0.333$ \\
\hline & LMF & $253.730 \pm 24.120$ & $0.529 \pm 0.050$ & $1.337 \pm 0.292$ & $3.357 \pm 0.059$ & $19.179 \pm 0.115$ \\
\hline \multirow{2}{*}{ CTR1 } & ATM & $13.250 \pm 3.150$ & $0.166 \pm 0.040$ & $3.567 \pm 0.419$ & $2.360 \pm 0.190$ & $18.227 \pm 2.025$ \\
\hline & LMF & $352.550 \pm 50.17$ & $0.734 \pm 0.105$ & $0.680 \pm 0.072$ & $2.400 \pm 0.020$ & $19.186 \pm 2.408$ \\
\hline
\end{tabular}

NB: Results are mean \pm standard deviation. Key: The amount of drug absorbed into the systemic circulation (D) calculated based on standard dose ( $45 \mathrm{Kg}$ /tablet dosing); bioavailability (F); absorption rate constant (Ka); maximum plasma concentration achieved (Cmax); a measure of total amount of unaltered drug that reaches the systemic circulation (AUC),starch (STC), sunflower oil (SFO), albumin (ALB), carbonated drinks (Cocacola ${ }^{\circledR} /$ Fanta $\left.{ }^{\circledR}\right)(\mathrm{CBS})$, lamivudine (LMV), metronidazole (MTN) and the control (CTR1).

\section{In vivo study}

The plasma concentration versus time plots of the dosing conditions in the in vivo experiments for ATM and LMF absorption are presented in Figures 4-7. Figure 4 showed that ATM absorption in the presence of fruit resulted in a higher $\mathrm{C}_{\max }(1.400 \mu \mathrm{g} / \mathrm{mL})$ than CTR2 $(0.867 \mu \mathrm{g} / \mathrm{mL})$ while there was no significant difference for LMF. Figure 4 representing EMS revealed an abysmally low ATM pharmacokinetic profile (Ka, $\mathrm{C}_{\max }$ and AUC) compared with CRT2 $(p<0.05)$ while LMF parameters did not show differences. Figure 5 showed the absorption characteristics for PMA and CTR2.
The individual plasma concentrations versus time profiles for the drug in each group computed showed large intra-group variability for EMS, FTS and CTR2 compared with the variability observed in the other treatments. However, there were no outliers (i.e., 10-fold unexplained deviation around data points) in the values for the subjects in each group for all the parameters. Figure 6 revealed the drug plasma concentration versus time profile for the regimen involving co-administration of $\mathrm{AL}$ with LMV and MTN. The pharmacokinetic profiles of AL under the respective regimens for in vivo experiments are summarized in Table 3.

Table 3: Pharmacokinetic profile for artemether and lumefantrine in the different regimen.

\begin{tabular}{|c|c|c|c|c|c|c|}
\hline Dosing regimen & Drugs analyzed & $\mathrm{D}(\mathrm{mg})$ & $\mathrm{F}$ & $\mathrm{Ka}\left(\mathrm{h}^{-1}\right)$ & $\mathrm{C}_{\max }(\mu \mathrm{g} / \mathrm{mL})$ & AUC $(\mu \mathrm{g} \cdot \mathrm{h} / \mathrm{mL})$ \\
\hline \multirow{2}{*}{ FTS } & Artemether & $39.151 \pm 0.729$ & $0.489 \pm 0.009$ & $0.536 \pm 0.061$ & $1.400 \pm 0.034$ & $8.797 \pm 0.119$ \\
\hline & Lumefantrine & $42.420 \pm 2.670$ & $0.088 \pm 0.005$ & $0.733 \pm 0.038$ & $2.000 \pm 0.114$ & $6.247 \pm 0.075$ \\
\hline \multirow{2}{*}{ EMS } & Artemether & $2.058 \pm 0.557$ & $0.026 \pm 0.007$ & $0.041 \pm 0.005$ & $0.117 \pm 0.028$ & $0.775 \pm 0.067$ \\
\hline & Lumefantrine & $196.30 \pm 8.030$ & $0.408 \pm 0.017$ & $0.659 \pm 0.025$ & $1.750 \pm 0.045$ & $14.375 \pm 0.234$ \\
\hline \multirow{2}{*}{ PMA } & Artemether & $66.501 \pm 5.353$ & $0.831 \pm 0.671$ & $0.435 \pm 0.019$ & $1.123 \pm 0.044$ & $12.805 \pm 0.548$ \\
\hline & Lumefantrine & $380.260 \pm 19.970$ & $0.792 \pm 0.042$ & $1.202 \pm 0.048$ & $3.243 \pm 0.038$ & $23.920 \pm 0.161$ \\
\hline \multirow{2}{*}{ CBS } & Artemether & $1.418 \pm 0.199$ & $0.018 \pm 0.003$ & $0.248 \pm 0.026$ & $0.643 \pm 0.104$ & $4.155 \pm 0.077$ \\
\hline & Lumefantrine & $51.030 \pm 3.300$ & $0.106 \pm 0.007$ & $0.912 \pm 0.060$ & $2.428 \pm 0.173$ & $8.722 \pm 0.164$ \\
\hline \multirow{2}{*}{ LMV } & Artemether & $45.360 \pm 3.396$ & $0.567 \pm 0.042$ & $0.342 \pm 0.031$ & $0.768 \pm 0.061$ & $6.193 \pm 0.077$ \\
\hline & Lumefantrine & $113.960 \pm 17.140$ & $0.237 \pm 0.035$ & $0.943 \pm 0.033$ & $2.495 \pm 0.202$ & $18.355 \pm 0.289$ \\
\hline \multirow{2}{*}{ MTN } & Artemether & $41.687 \pm 1.879$ & $0.527 \pm 0.024$ & $0.448 \pm 0.035$ & $1.063 \pm 0.025$ & $6.873 \pm 0.066$ \\
\hline & Lumefantrine & $289.830 \pm 6.950$ & $0.604 \pm 0.014$ & $1.165 \pm 0.060$ & $2.823 \pm 0.129$ & $22.312 \pm 0.842$ \\
\hline \multirow{2}{*}{ CTR2 } & Artemether & $42.782 \pm 1.856$ & $0.535 \pm 0.023$ & $0.362 \pm 0.082$ & $0.867 \pm 0.038$ & $6.090 \pm 0.113$ \\
\hline & Lumefantrine & $317.480 \pm 5.310$ & $0.661 \pm 0.011$ & $1.109 \pm 0.006$ & $2.798 \pm 0.069$ & $18.878 \pm 0.162$ \\
\hline
\end{tabular}

NB: Results are mean \pm standard deviation. Key: $\mathrm{D}, \mathrm{F} \mathrm{Ka}, \mathrm{C}_{\max }$ and $\mathrm{AUC}$ represent total amount of drug absorbed into the body, absolutebioavailability, absorption rate constant, maximum plasma concentration and area under the curve, respectively. EMS is "eba" with melon soup, PMA represents pap with milk and "akara", CBS is carbonated drinks (Cocacola ${ }^{\circledR} /$ Fanta $\left.{ }^{\circledR}\right)$, LMV is lamivudine, MTN is metronidazole and CTR2 is fasted group serving as control. 

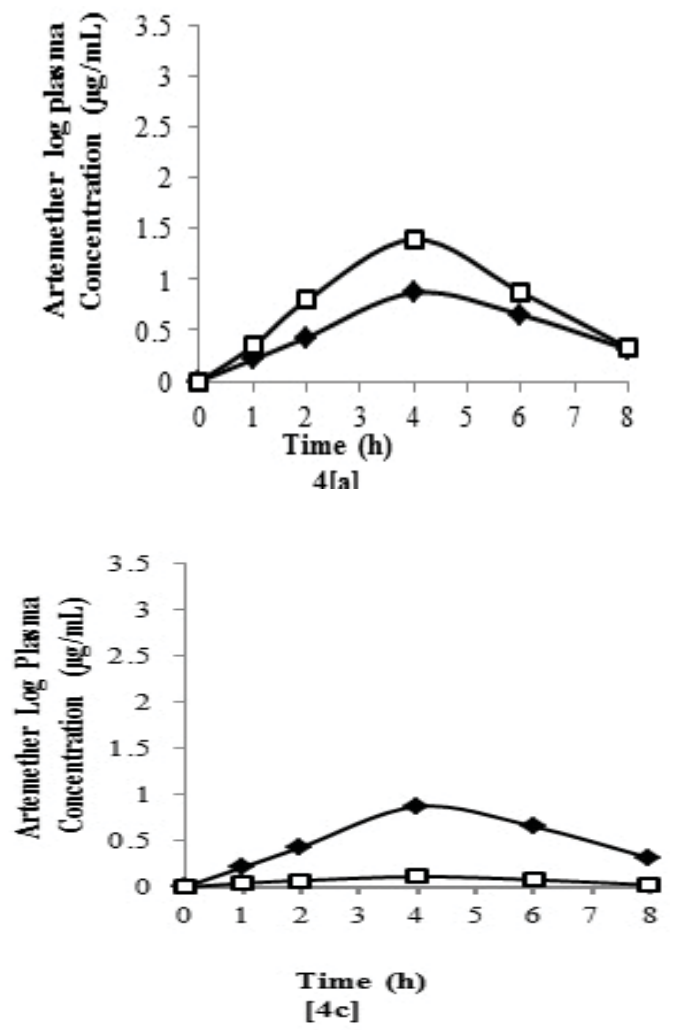
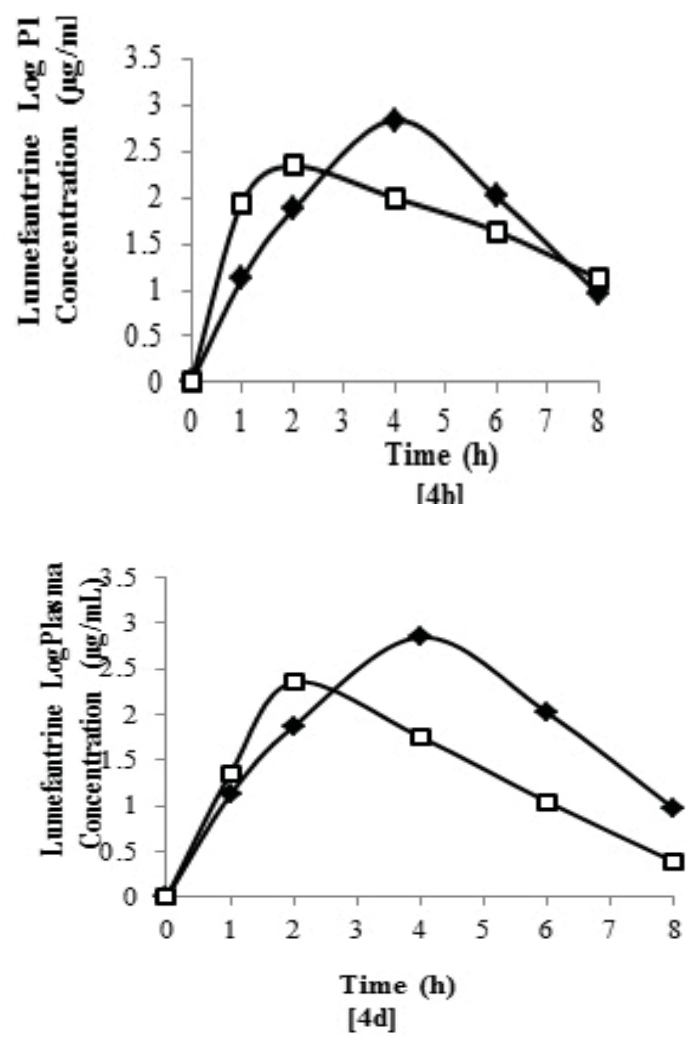

Figure 4: Drug plasma concentration versus time plot for AL from tablets co-administered with FTS or EMS; 4a and 4b: ( $\bullet$ CTR2 and ( $\square$ ) FTS while 4c and 4d: $(\diamond)$ CTR2 and (口) EMS.

The types of meals taken with antimalarial drugs especially in the tropics vary widely and there is the need to evaluate the bioavailability implications. Several studies have focused on antimalarial drug effectiveness which span from the quality profiling to formulating convenient dosage forms to achieve better patient compliance and ultimately therapeutic efficacy ${ }^{[18,19,22]}$. This present study points out the effects of some meals, beverages alongside selected drugs on the pharmacokinetic disposition of the actives in AL tablets. The in vitro food-drug interaction between food components and AL has been reported in an earlier study ${ }^{[23]}$. The goal was to carry out a preliminary in vitro investigation into the mechanisms of food and $\mathrm{AL}$ interaction if any ${ }^{[23]}$. There are however scanty data relating to the absorption and disposition of AL that may influence AL treatment outcome. This study therefore, assessed the oral absorption of the component drugs of AL in the light of real life meal types and commonly co-prescribed drugs. It also provides explanation for the physicochemical basis and relationship between the composition of food types and the investigated drugs with the actives in AL tablets, on co-administration

The result obtained in the in situ model projected the recommendations of the manufacturer of AL that the drug should be taken with a fatty meal ${ }^{[24]}$. SFO gave the optimum absorption for AL as seen in ATM and LMF dispositions. SFO produced optimum absorption and bioavailability for the actives in AL tablets.

An earlier study by Premji and co-workers reported that African meals contained enough fat for LMF oral absorption $^{[17]}$. It suffices to state that a wide array of diets taken in real life malarial conditions may have little or no fat at all. Arinze-Ovia and co-workers in South Eastern Nigeria, reported that oily diets were culturally restricted during malarial episodes based on the wide acceptance of the norm that oil contributes to the incidence of malaria ${ }^{[17]}$. Unfortunately this will lead to a reduced absorption of ATM and in particular LMF as shown in this study. Furthermore, STC reduced the oral absorption of ATM and LMF significantly compared with CRT1 and SFO. Morais and co-workers reported on the roles of resistant and digestible STC on drug absorption ${ }^{[25]}$. High fibre foods have also been reported to demonstrate unpredictable effects on medication absorption. Insoluble dietary fibre found in bran or brown rice was reported to inhibit digoxin absorption $^{[26]}$. We propose a mechanism for STC reduction of ATM and LMF absorption as the formation of sticky gel in the presence of water and consequently increase in viscosity of the absorbing medium, thereby reducing molecular mobility and therefore rate/extent of absorption. 
This argument is in line with the thoughts of Cichero and co-workers ${ }^{[27]}$ who projected the effects of thickening agents on stomach fill and satiety.

This in situ experiment also revealed that ALB caused a significant reduction in the absorption of ATM and LMF. There have been reports that the bioactive ingredients in protein-rich foods bind to certain drugs leading to reduction in drug absorption ${ }^{[28]}$. In the study area, malarial patients may take chicken, fish or meat-rich soups as appetizers prior to ingestion of malaria drugs. The result in this study presents the possible outcome of taking AL with proteinrich diet. Furthermore, the in situ experiment revealed that CBS treatment led to significantly reduced oral absorption of the actives of AL tablets compared with CRT1.The presence of CBS has been reported to cause significant alteration of gastrointestinal $\mathrm{pH}^{[23]}$. Administering CBS therefore will favour the solubility and dissolution of acidic drugs in the gastrum. ATM and LMF are liphophilic drugs and are essentially absorbed in the intestine. The acidic disposition of CBS therefore may only retard the solubility of AL. An earlier study revealed that CBS forms complexes with the actives in AL and this may pose a greater hitch to the absorption of AL in the intestine ${ }^{[23]}$.

This study also revealed that MTN and LMV caused significant reduction in the oral absorption of ATM and LMF. LMV and MTN have been reported to cause pancreatitis manifesting with nausea and diarrhea. The concomitant use of AL with these drugs and the consequent increase in gastric motility may reduce the absorption and bioavailability. Furthermore drug dissolution is important for drug absorption; the dehydration consequent to diarrhea may cause reduction in the rate of drug dissolution, seriously affecting drug bioavailability.

The meals employed in the human experiment were selected based on the widely embraced diet types across the geo-cultural boundaries in Southern Nigeria and its environs, especially during episodes of malaria. Comparing the meals in Nigeria with the Food and Drug Administration recommended standard fatty meal; the meals in the former differed significantly with respect to composition and volume ${ }^{[24]}$. It contained more fat and may pose a better milieu for AL dissolution. This study revealed striking differences in the pharmacokinetic parameters for the different regimens employed.

FTS did not cause significant changes in the pharmacokinetic parameters of ATM and LMF compared with CRT2. FTS contains simple sugars that required little or no further digestion before it is passively absorbed; therefore co-ingestion with AL may not alter the absorption of drugs unless there is competitive mechanisms of absorption at the absorptive surfaces. As the duodenal surface is extensive, the presence of a co-absorbing molecule may by a stoichiometric relationship which significantly impact on the absorption of one entity due to the other. Unlike in the in situ experiment, FTS differed from STC which presented a different outcome on AL absorption as this consists of polysaccharides that swells in water resulting in increasing viscosity. EMS meal however, produced a result akin to STC as it is made up of polysaccharide, like STC. EMS however consists of oily melon soup that gives a scenario of fatty milieu recommended for AL absorption. However, the pharmacokinetic disposition of ATM and LMF was inhibited by this meal type compared to the values of the respective parameters in CRT2.

The study revealed that PMA produced significantly higher pharmacokinetic parameters for AL than observed for CRT2. The milk and oil-fried "akara" contained oil that can enhance the dissolution of lipophilic drugs. However, some studies have revealed that food such as milk and its casein content decrease the absorption of drugs like ciprofloxacin ${ }^{[29]}$ and norfloxacin ${ }^{[30]}$ but another study concluded milk as not influencing the absorption of a structurally related fluoroquinolone antibacterial, ofloxacin $^{[31]}$. PMA meal is a common food in Southern Nigerian and Western African dishes featuring milk and its constituent divalent ions (i.e, calcium) which has been implicated in chelating effects on drugs especially the antibacterial agents ${ }^{[30]}$. In this experiment, PMA revealed significantly higher pharmacokinetic parameters for ATM compared with CRT2 (Figure 3). ATM being a lipophilic drug is believed to have presented enhanced absorption indices in the presence of milk because of the fat content.

Carbonated beverages (e.g., Cocacola ${ }^{\circledR}$ and Fanta ${ }^{\circledR}$ ) are acidic beverages and may present an acidic environment in the gastrum thereby affecting the extent of ionization (the ratio of ionized to unionized molecules) of a co-ingested drug. The $\mathrm{pH}$ in the stomach has been extensively reported to affect the absorption of drugs ${ }^{[32]}$. An earlier study showed high acid buffering capacities in the stomach by these widely consumed beverages ${ }^{[24]}$. It also revealed that the complexation potentials of constituents of the beverages with AL were characterized by the marked changes in spectral features of AL on co-pelletization with Cocacola ${ }^{\circledR}$. The mechanism of pharmacokinetic interactions of acidic beverages with $\mathrm{AL}$, in vivo is presumed to be physiological and involves a chelation/complexation of molecules involved, in the light of these studies. LMV did not alter the bioavailability of ATM but significantly reduced that of LMF $(p<0.05)$. 

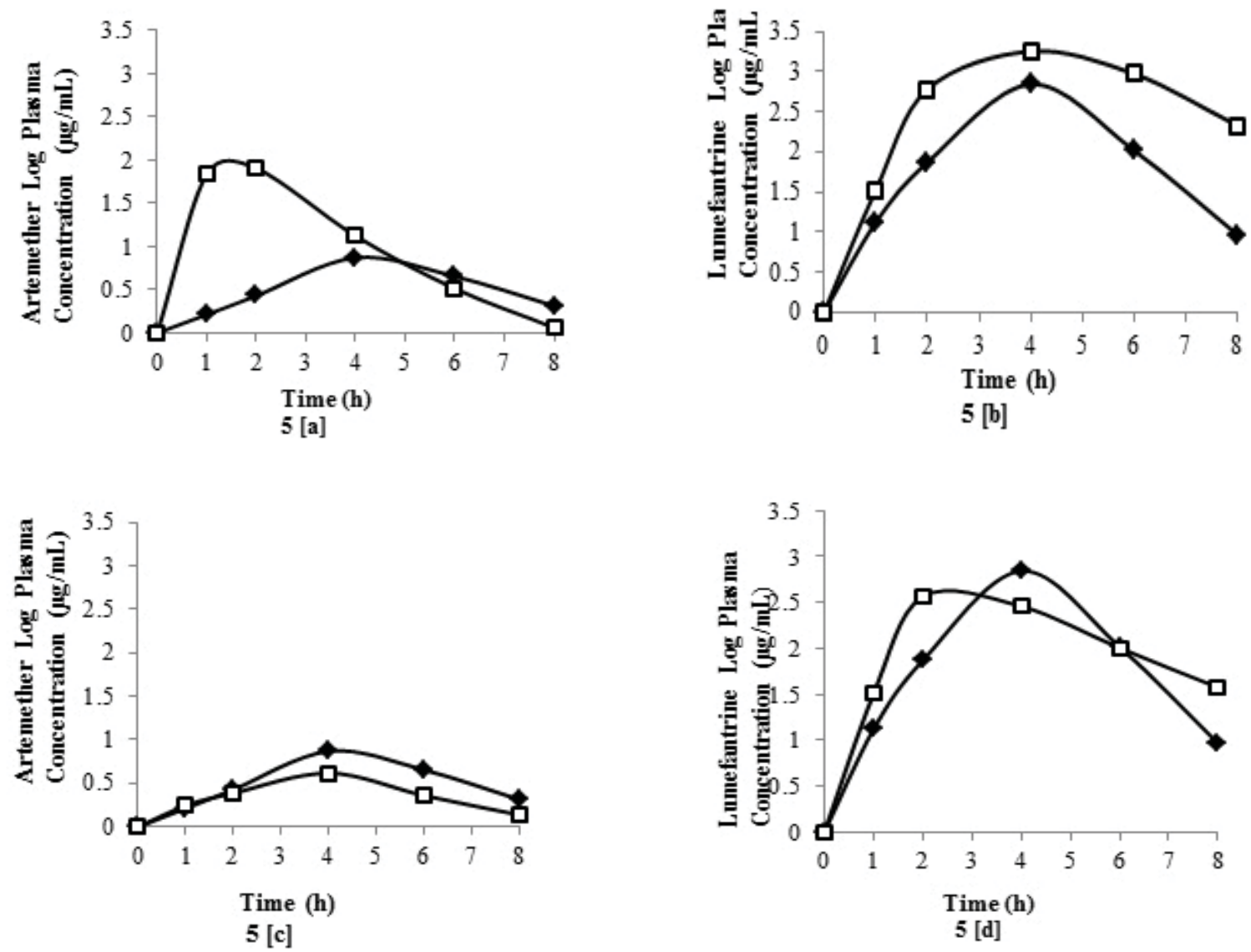

Figure 5: Drug plasma concentration versus time plot for AL from tablets co-administered with PMA and CBS; 5a and 5b: ( $\downarrow$ ) CTR2 and ( $\square$ ) PMA while 5c and 5d: $(\bullet)$ CTR2 and ( $\square$ ) CBS.

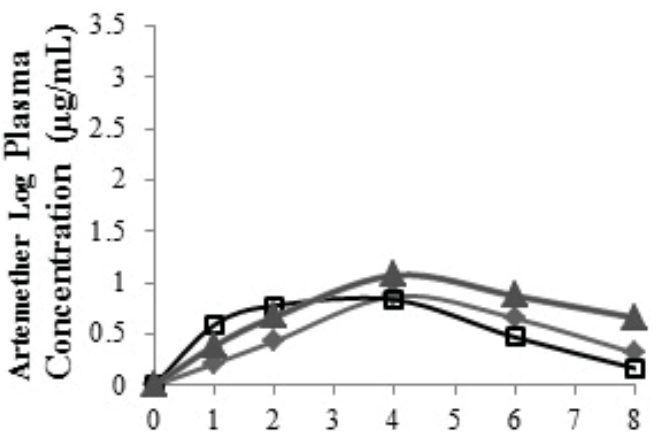

Time (h)

[a]

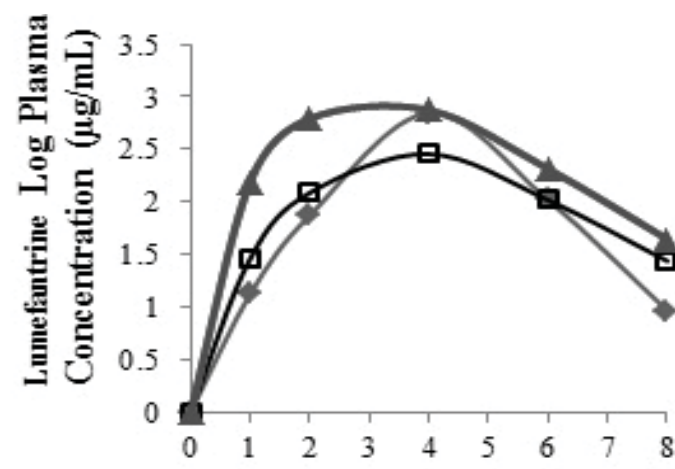

Time (h)

Figure 6: Drug plasma concentration versus time plot for AL from tablets co-administered with drugs in vivo; (४) CTR2, ( $\square)$ LMV and ( $\Delta$ ) MTN. CTR2 and ( $\square$ ) PMA while 5c and 5d: ( ) CTR2 and ( $\square$ ) CBS. 
The in situ experiment also corroborated the effect of LMV on LMF absorption. Byakika-Kibwika et al reported on the significant influence of lopinavir/ritonavir on pharmacokinetic exposure of AL in HIV-infected Ugandan adults ${ }^{[9]}$. Drug-drug interaction can be expected when an antiretroviral drug is co-administered with antimalarial agent. There is however scanty information in the literature on drug-drug interaction of LMV on co-administration with other drugs. Similarly, the effect of MTN was on LMF absorption as it reduced the bioavailability of LMF. MTN and LMV are drugs that are known to increase gastrointestinal motility thereby increasing the transit time of co-administered drugs. They have been reported to cause nausea and vomiting ${ }^{[33,34]}$. The speed up passage of drugs by MTN and LMV through the absorptive environment may inhibit drug dissolution and thereby affect absorption ${ }^{[35]}$.

\section{CONCLUSIONS}

The absorption of ATM and LMF varied widely within the different meal types. There was significant reduction in the total drug absorbed for EMS and CBS compared with fasted regimen while PMA produced enhanced absorption for both ATM and LMF. There was a reasonable correlation among data obtained from in situ model and those obtained from the in vivo model.

\section{ACKNOWLEDGEMENTS}

The authors are grateful to Dominic Nkanang Ekaete of University of Uyo Health Centre for coordinating the sample collection and Peter Douglas Ojobor of University of Lagos Central Research Laboratory for his technical assistance.

\section{CONFLICT OF INTEREST}

The authors declare no conflict of interest.

FUND

The authors declare that the funding from TetFund Merged Intervention 2015-2016 given to Sunday Olajide Awofisayo was used for this study.

\section{REFERENCES}

1. K.I. Barnes, P. Chanda, G. Barnabas, Impact of the large scale deployment of artemether-lumefantrine in the malaria disease burden in Africa: case studies of South Africa, Zambia and Ethiopia, Malar. J., 8 (Suppl.) (2009) S8.

2. A.Y. Maugmu, K.B.H. Hassan, Malaria as a cause of morbidity and mortality: A socio-economic overview, Res. Human Soc. Sci., 5 (2015) 100105.

3. World Health Organization, Malaria fact sheet. World Health Organization, Geneva, Switzerland, 2013.
4. A. G. Djimde, G. Lefevre, Understanding the pharmacokinetics of Coartem. Malar. J., 8(2009) S4-S9.

5. C. Chen, Development of antimalarial drugs and their application in China: a historical perspective, Infect. Dis. Pov., 3 (2014) 1-10.

6. S. Abdulla, I. Sagara, S. Borrman, U. D’Alessandro, R. Gonzalez, M. Hamel, Efficacy and Safety of artemether-lumefantrine dispersible tablet compared with crushed commercial tablets in African infants and children with uncomplicated malaria: a randomized, single-blind, multicentre trial, Lancet, 372 (2008) 1819-1827.

7. S. Abdulla, I. Sagara, Dispersible formulation of artemether/lumefantrine: specifically developed for infants and young children, Malar. J., 8 (2009) 1 article S7.

8. P. Byakika-Kibwika, M. Lamorde, V. OkabaKayom, H. Mayanja-Kizza, Significant pharmacokinetic interaction between artemether/ lumefantrine and efavirenz in HIV-infected Ugandan Adults, J. Antimicrob. Chemother., 67( 2002) 2213-2221.

9. D.S. Karuna, A.K. Rasheedhabanu, U. Ubaidulla, G. Rathnam, Development of artemether and lumefantrine fixed dose combination tablets: Preparation, characterization and in-vitro evaluation, Int. J. Pharm., 4 (2014) 166-172.

10. World Health Organization, Assessment of therapeutic efficacy of antimalarial drugs for uncomplicated P. falciparum malaria in areas with intense transmission, WHO, Geneva, (2003) 5-12.

11. World Health Organization, Guidelines for the treatment of malaria, $2^{\text {nd }}$ Ed. Geneva, Switzerland, (2010) http://www.who.int/malaria/publication/ atoz/9789241542928/en/index.html.

12. C. Cohen, A. Karstaedt, J. Frean, J. Thomas, N. Govender, E. Prentice, L. Din, J. Galpin, H. CreveBrown, 2005. Increased prevalence of severe malaria in HIV-infected adults in South Africa, Clin. Infect. Dis., 41 (2005)1631-1637.

13. P. Byakika-Kibwika, M. Lamorde, H. MayanjaKizza, S. Khoo, C. Merry, J. van Geertruyden, Artemether-lumefantrine combination therapy for treatment of uncomplicated malaria: the potentials for complex interaction with anti-retroviral drugs in HIV-infected individuals, Malar. Res. Treat. (2011) Article ID 703730. http:// dx.doi. org/10.4061/2011/703730. 
14. World Health Organization, Malaria and HIV and their implications for public policy. World Health Organization, Geneva, Switzerland, (2005).

15. W. Huang, S.L. Lee, L.X. Yu, Mechanistic approaches to predicting oral drug absorption, Am. Assoc. Pharm. Sci. J., 2 (2009) 217-224.

16. S.U. Arinze-Onyia, U. Ugwoke, E.N Aguwa, I. Modebe, E.D. Nwobodo, A. Ilika, Myths and facts on malaria. A pilot study of community oriented resource persons in rural communities of Anambra, Southeast Nigeria, Int. Res. J. Med. Sci., 2 (2014) 91-97.

17. Z.G. Premji, S. Abdua, B. Ogutu, A. Ndung, C. O Falade, I. Sagara, The content of fat in African diets is adequate to achieve optimal efficacy with fixed-dose artemether-lumefantrine: A review of the evidence, Malar. J., 7 (2008) 244-51.

18. P.G. Welling, Effect of food on drug absorption. Ann. Rev. Nutr., 16 (2013) 383-415.

19. T. Kredo, K. Mauff, J.S. Van der Walt, L. Wiesner, G. Maarlens, K. Cohen, P. Smith, K.I. Barnes, Interaction between artemether-lumefantrine and nevirapine-based antiretroviral therapy in HIV-1 infected patients, Antimicrob. Agents Chemother., 55 (2011)5616-23.

20. N. Mwebaza, M Jerling, L.L. Gustafsson, C. Obua, P. Waako, M. Mahindi, M. Ntale, O. Beck, U. Hellgren, Comparable lumefantrine oral bioavailability when co-administered with oil-fortified maize porridge or milk in healthy volunteers, Basic Clin. Pharmacol Toxicol. 113(2013)66-72.

21. American Medical Association (AMA), World Medical Declaration of Helsinki Ethical Principles for Medical Research involving Human Subjects, J. Am. Med. Assoc., 310 (2013) 2191-4.

22. S.O. Awofisayo, E. Willie, E. Umoh, Quality control evaluation of multi-source artemetherlumefantrine tablets prescribed for uncomplicated multi-drug resistant malaria, Ind. J. Novel Drug Deliv., 2(2010) 153-157.

23. S.O. Awofisayo, M.I. Arhewoh, A.O. Okhamafe, In vitro interaction of food component with $\mathrm{AL}$ tablet, Int. J. Curr. Res. Rev., 10(2018) 22-26.
24. A. Djimde, G. Lefevre, Understanding the pharmacokinetics of Coartem, Malar. J., 12 (2009) 8 Suppl. 1: S4. Doi: 10.1186/1475-2875-8-S1-S4.

25. M.B. Morais, A. Feste, R. G. Miller, C. H. Lifschitz., Effect of resistant and digestible starch in intestinal absorption of calcium, iron and zinc in infant pigs, Pediatr Res. 39(1996) 872-6.

26. A.G. Canga, N.F. Martinez, A. M. Sahagun Prieto, J. J. Garcia Vietez, M. J. DiezLiebana, R. DiezLaiz, M. Sierra Vega, Dietary fiber and its interaction with drugs, Nutr. Hosp., 25(2010) 535-539.

27. J.A.Y. Cichero, Thickening agents used for dysphagia management: effect on bioavailability of water, medication and feelings of satiety, Nutr. J., 12 (2013) 54-63.

28. R. Bushra, N. Aslam, A.Y. Khan, Food-Drug Interactions, Oman Med. J., 26 (2011) 77-83.

29. P.J. Neuvonen, M. N. Niemi, J.T Backman, Drug interactions with lipid lowering drugs: Mechanisms and clinical relevance, Clin. Pharmacol. Ther., 80 (2006) 565-581.

30. K.T. Kivisto, P. Ojala-Karlsson, P. J. Neuvonen, Inhibition of norfloxacin absorption by dairy products, Antimicrob. Agents Chemother., 36(1992) 489-91.

31. P.J. Neuvonen, K. T. Kivisto, Milk and yoghurt do not impair the absorption of ofloxacin, Br. J. Clin. Pharmacol., 33(1992) 346-348.

32. A. Mitra, F. Kesisoghou, Impaired drug absorption due to high stomach $\mathrm{pH}$ : a review of strategies for mitigation of such effect to enable pharmaceutical product development, Mol. Pharm., 10(2013) 3970-9.

33. S.O. Awofisayo., M. I. Arhewoh, A. O. Okhamafe, In-vitro interaction studies between artemetherlumefantrine and lamivudine/metronidazole, Int. J. Curr. Res. Rev., 12(2018) 1-6.

34. E. O'Halloran, A. Hogan, K. Mealy, Metronidazole induced pancreatitis. HPB Surgery, (2010) Article ID 523468, 4 pages. Doi.org/10.1155/2010/523468.

35. M.B. Kaufman, Drug-induced pancreatitis., Pharm. Ther., 38(2013) 349-351. 